\title{
Giant Urinary Bladder Stone in a Middle-Aged Male
}

\author{
Authors: \\ João Cleber Coutinho Pires, ${ }^{1}$ Breno Bittencourt de Brito, ${ }^{2}$ Fabrício \\ Freire de Melo, ${ }^{2}{ }^{*}$ Cláudio Lima Souza, ${ }^{2}$ Márcio Vasconcelos Oliveira ${ }^{2}$ \\ 1. Hospital Municipal Antônio Teixeira Sobrinho, Jacobina, Bahia, Brazil \\ 2. Instituto Multidisciplinar em Saúde, Universidade Federal da Bahia, Vitória da \\ Conquista, Bahia, Brazil \\ *Correspondence to caulimas@gmail.com \\ Disclosure: \\ The authors have declared no conflicts of interest. \\ Received: \\ 04.11.21 \\ Accepted: \\ 15.12 .21 \\ Keywords: \\ Cystolithotomy, giant bladder stone, haematuria, urinary symptoms, urolithiasis. \\ Citation: \\ EMJ Urol. 2022; DOI/10.33590/emjurol/21-00239
}

\begin{abstract}
Urolithiasis is a disorder that affects $10-15 \%$ of people at least once in their lives. Among the calculi affecting the urinary tract are the bladder stones. Giant urinary bladder stones are rare and can potentially lead to the onset of intense symptoms as well as life threatening repercussions. This disorder demands an accurate diagnosis with the use of imaging tools and laboratory tests, as well as an agile and appropriate therapeutic approach in order to prevent unfavourable outcomes. This paper reports on a bladder stone that was $18.5 \mathrm{~cm}$ in diameter and weighed $1.328 \mathrm{~kg}$, which was extracted from a middle-aged male.
\end{abstract}

\section{INTRODUCTION}

Approximately $10-15 \%$ of the general population is affected by urolithiases at least once in their lifetime.' Having a giant urinary bladder stone is a particularly rare condition and its development is usually associated with disorders that promote urinary stasis. ${ }^{2}$ This type of urolith can lead to serious health repercussions, including hydronephrosis and kidney failure. ${ }^{3}$ However, despite being a life threatening urological disease, scientific evidence on this issue remains sparse. Here, the authors report the occurrence of a bladder stone that was $18.5 \mathrm{~cm}$ in diameter and weighed $1.328 \mathrm{~kg}$ in a middle-aged male. To the authors' knowledge, this is the bladder stone with the largest diameter reported to date. Written informed consent was obtained from the patient for publication of this case report and any accompanying images.

\section{CASE REPORT}

The case was a 51-year-old male patient with no previous relevant medical history, who attended to a medical consultation on $22^{\text {nd }}$ March 2019, complaining of hypogastric pain and intermittent haematuria. He had no history of urinary tract infections, previous surgeries, or any factor suggesting neurogenic bladder, benign prostatic hyperplasia, and urethral stricture. On physical examination, he had a firm, mobile, hypogastric mass. Serum analysis revealed mild normocytic and normochromic anaemia (haemoglobin: 12.6 $\mathrm{g} / \mathrm{dL}$ ). Moreover, urinalysis evidenced slightly cloudy urine, proteinuria (1+), leukocyturia (2+), ketonuria (1+), haemoglobinuria (2+), pyuria (20 piocytes/field), haematuria (18 red blood cells/ field), and rare epithelial cells. No bacterial growth was observed in the final culture. Ultrasound imaging (USI) of the abdomen demonstrated 
a large mass inside the urinary bladder, with posterior acoustic shadowing, suggesting a giant bladder stone. The USI also evidenced marked hydronephrosis on the right (Grade III/IV) and mild hydronephrosis on the left (Grade I/IV) as well as bilateral proximal ureteral dilatations. The sizes of the prostate and its medial lobe were not increased. The residual post-voiding volume was $184.5 \mathrm{~mL}$ (Figure 1). Nine weeks later, the patient underwent a cystolithotomy, with removal of a stone measuring $185 \mathrm{~mm}$ at its largest diameter and weighing $1.328 \mathrm{~kg}$ (Figure 2). The surgery was performed through an $18 \mathrm{~cm}$ infraumbilical incision due to the stone size. There was no insertion of a suprapubic catheter and no retrograde cystogram was available. Urethral catheterisation was performed. On the second postoperative day, he underwent an abdominal CT for an ileus investigation and to discard the possibility of intestinal perforation. The CT evidenced a urinary bladder with enlarged dimensions as well as a diffuse, remarkable wall thickening. In addition, a heterogeneous material inside the bladder was observed, originating iso/ hypoattenuating images suggesting gaseous foci and small stones (at least five stones smaller than $0.3 \mathrm{~cm}$ and one with $0.5 \times 0.5 \times 0.7 \mathrm{~cm}$ ). A minor ectasia was found in the right pyelocaliceal system. The patient had a satisfactory recovery with no further complaints, and he was discharged on the fifth postoperative day.

\section{DISCUSSION}

Bladder stones represent approximately 5\% of urinary tract calculi, and giant bladder stones account for only $1 \%$ of urolithiases. ${ }^{4,5}$ Of note, the incidence of vesical calculi is higher in developing countries and this condition is responsible for $8 \%$ of urolithiasis-related deaths. ${ }^{6}$ This phenomenon occurs mainly among males, who represent 95\% of cases. ${ }^{7}$ Bladder stones can be classified into primary and secondary. The former develops without any known abnormality that facilitates stone formation. The latter are preceded by a recognisable aetiologic cause. Disorders favouring urinary stasis and, consequently, the precipitation of calcium, oxalate, phosphate, and uric acid predispose the formation of bladder stones. Among these conditions, benign prostatic hyperplasia, urethral stricture, and neurogenic bladder stand out.8

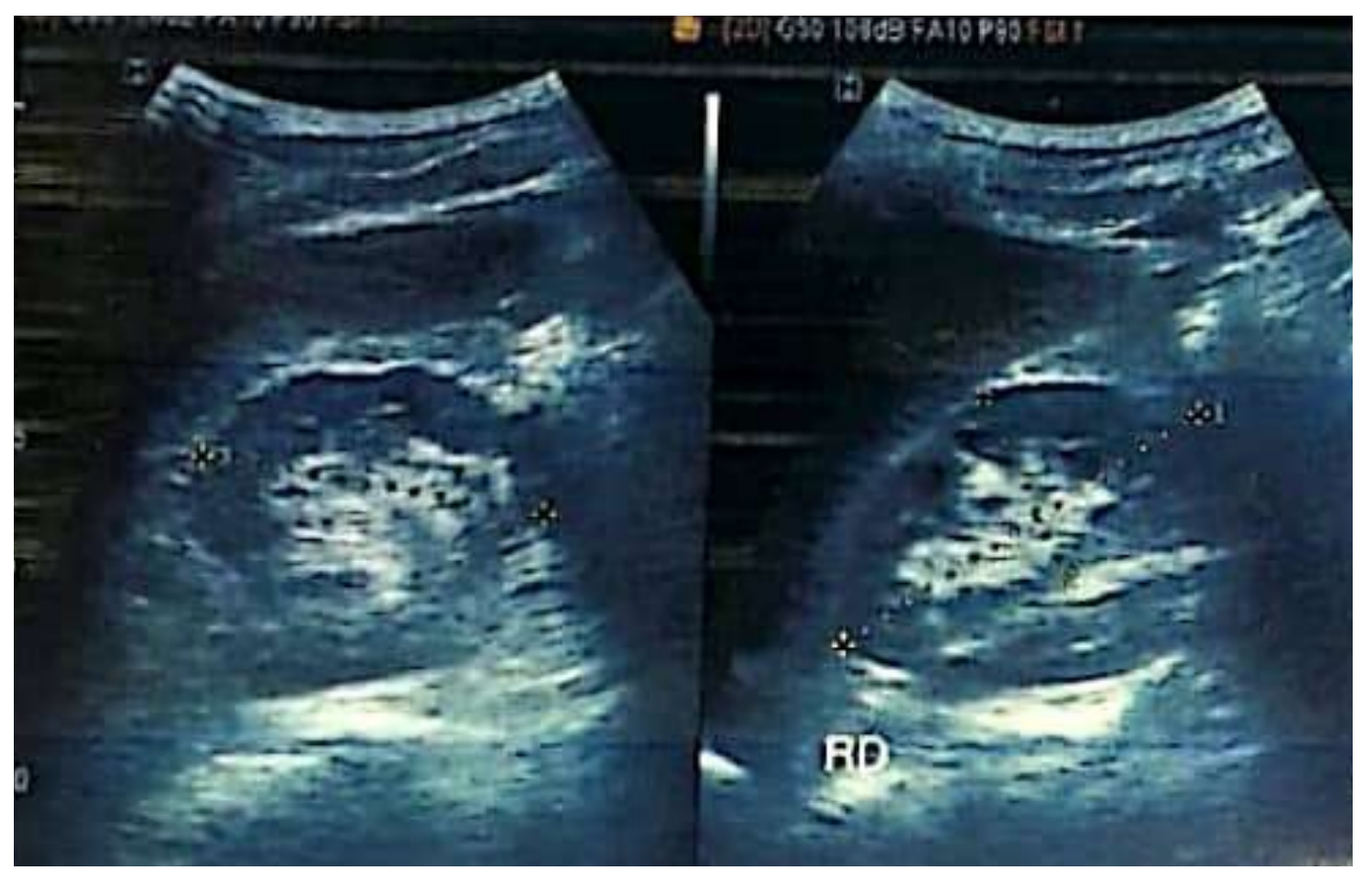

Figure 1: Ultrasound images of the urinary bladder with the giant bladder stone inside, which is represented by a hyperechoic image with acoustic shadowing. 

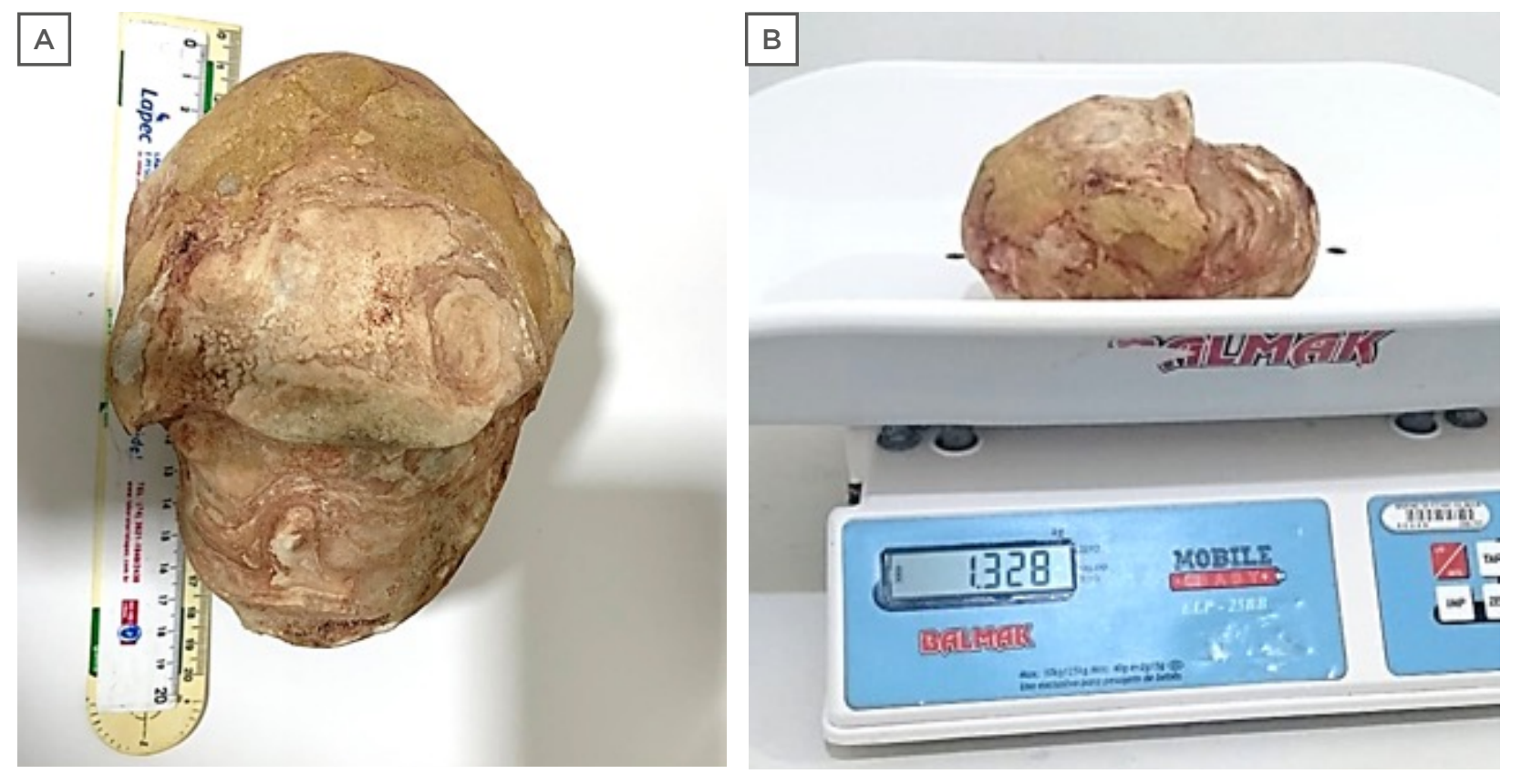

Figure 2: Pictures demonstrating the $(A)$ diameter $(18.5 \mathrm{~cm})$ and $(B)$ weight $(1.328 \mathrm{~kg})$ of the giant bladder stone removed from the patient.

Besides male gender, no other risk factor for the occurrence of bladder stones was identified in the authors' patient, who probably had a primary bladder stone. Of note, the occurrence of primary bladder stones has been associated with low socioeconomic status and cerealbased diets, which may lead to metabolic repercussions including increased acid uric levels, hypophosphaturia, and hyperammoniuria. ${ }^{9}$

Regarding chemical characteristics, most bladder stones have mixed composition, and these properties are influenced by $\mathrm{pH}$, degree of urine saturation, and possible micro-organisms in the urine. Bladder calculi are mostly made up of uric acid and urate. The presence of Proteus in the urine has been associated with calcium phosphate and struvite stones, whereas Escherichia coli has been related to the occurrence of calcium oxalate and urate calculi. ${ }^{6}$ Unfortunately, the study of stone composition was not performed in the case reported here.

Complaints of occasional haematuria and suprapubic pain are often observed in individuals with bladder stones, and they were present in the aforementioned case. Furthermore, patients frequently refer to urinary symptoms and urinary urgency. ${ }^{10,11}$ USI of the bladder is considered the first choice for bladder stone diagnosis due to its broad availability, whereas cystoscopy has the highest accuracy in the detection of this disorder since the former may not distinguish bladder calculi from tumours on some occasions. The calculi may also be detected through X-rays, but $50 \%$ of the bladder stones are radiolucent. ${ }^{22}$

A CT scan was performed in the authors' case for a post-surgical evaluation of an ileus, but this method is not often used as a diagnostic tool for bladder lithiasis as performing cheaper imaging methods is more advantageous in this clinical scenario." Since bacterial colonisation favours lithogenesis and bladder calculi predispose to infection, it is useful to perform a urine culture test, which did not verify bacterial growth in the authors' case.12 Hydroureter and bilateral hydronephrosis are rare outcomes in individuals with bladder lithiasis. ${ }^{13}$ The occurrence of these repercussions in the present case was probably due to the huge size of the calculus $(185 \mathrm{~mm}$ in diameter and $1.328 \mathrm{~kg}$ in weight), leading to a considerable obstruction of the urinary flow. The surgery was performed 9 weeks after the diagnosis due to difficulties making this procedure quickly available for patients in the Brazilian public health system. 
Previous reports on giant bladder stones available on PubMed were assessed by searching for "giant" AND "bladder" OR "vesical" AND "stone" OR "calculus" OR "calculi" in article titles and abstracts, and 184 articles were found. This investigation was not able to find any description of a bladder stone with a larger diameter than that observed in the case reported here $(185 \mathrm{~mm})$. Regarding weight, four articles reported heavier bladder calculi than the stone found in the present case (1,328 g), weighting $1,815 \mathrm{~g},{ }^{14} 1,640 \mathrm{~g},{ }^{15} 1,620 \mathrm{~g},{ }^{16}$ and $1,410 \mathrm{~g} \cdot{ }^{17}$ No other urinary dysfunction was associated with the first case report, ${ }^{15}$ whereas two of those bladder stones were associated with bladder carcinoma ${ }^{15,17}$ and one of them was related to a neurogenic bladder. ${ }^{16}$

Various methods are available for the removal of bladder stones, including less invasive procedures such as cystolithotomy, extracorporeal shock wave lithotripsy, and transurethral cystolithotripsy." Laparoscopic surgery is a good alternative, particularly in patients with solitary stones that are $3 \mathrm{~cm}$ or less. ${ }^{18}$ Robotic cystolithotomy has also been performed in this context, with promising results. ${ }^{19}$ However, the preferred procedure for the treatment of giant calculi is still the cystolithotomy, as observed in this case.

\section{CONCLUSION}

Here, the authors reported the urinary bladder stone with the largest diameter ever described. Giant vesical calculus is a rare but potentially life-threatening condition that can lead to symptoms that importantly impair the life quality of affected individuals. The available data on this disease are scarce, and this report describing such a huge calculus may contribute to a better understanding of this health problem.

\section{References}

1. Long LO, Park S. Update on nephrolithiasis management. Minerva Urol Nefrol. 2007;59(3):317-25.

2. Hızlı F, Yılmaz E. A giant bladder struvite stone in an adolescent boy. Urol Res. 2012;40(3):273-4.

3. Dhondup $T$ et al. Risk of ESRD and mortality in kidney and bladder stone formers. Am J Kidney Dis. 2018;72(6):790-7.

4. Hammad FT et al. Bladder calculi: did the clinical picture change? Urology. 2006;67(6):1154-8.

5. Rahman $\mathrm{M}$ et al. A giant vesical calculus. Mymensingh Med J. 2007;16(Suppl 2):S57-9.

6. European Association of Urology (EAU). Bladder stones. 2021. Available at: https://uroweb.org/guideline/ bladder-stones/\#4. Last accessed: 15 December 2021
7. Pomerantz PA. Giant vesical calculus formed around arterial graft incorporated into bladder. Urology. 1989;33(1):57-8.

8. Ishtiaq $\mathrm{R}$ et al. An uncommon cause of dysuria in a female patient: huge urinary bladder stones. Cureus. 2017;9(10):e1788

9. Teotia $\mathrm{M}$ et al. Fluoride metabolism and fluoride content of stones from children with endemic vesical stones. Br J Urol. 1991;68(4):425-9.

10. Vilar DG et al. [Giant bladder lithiasis: case report and bibliographic review]. Arch Esp Urol. 2011;64(4):383-7. (In Spanish).

11. Cicione A et al. Bladder stone management: an update. Minerva Urol Nefrol. 2018;70(1):53-65.

12. Miano $\mathrm{R}$ et al. Stones and urinary tract infections. Urol Int. 2007;79(Suppl 1):32-6.
13. Lameire $\mathrm{N}$ et al. Acute renal failure. Lancet. 2005;365(9457):417-30.

14. Randall A. Giant vesical calculus. J Urol. 1921;5:119-25.

15. Nyguard E, Terjesen T. Giant vesical cauculus and anuria. Scand J Urol Nephrol. 1976;10:88-90.

16. Ulukok C. [A monstrous bladder calculus]. Acrci Urol Belg. 1967;35(4):643-4. (In French).

17. Powers JH, Matflerd RG. Giant vesical calculus and carcinoma of the bladder. J Urol. 1952;67:184-91.

18. Batislam $E$ et al. A new application of laparoscopic instruments in percutaneous bladder stone removal. $\mathrm{J}$ Laparoendosc Adv Surg Tech A. 1997;7(4):241-4.

19. Esposito $\mathrm{C}$ et al. Minimally invasive management of bladder stones in children. Front Pediatr. 2021;8:618756. 